\title{
A Note on the Fuzzy Linear Maps over the Fuzzy Quotient Spaces
}

\author{
Chang Bum Kim* \\ Department of Mathematics, Kookmin University, Seoul 136-702, South Korea
}

\begin{abstract}
In this paper we study various properties of the fuzzy linear maps over the fuzzy quotient spaces. In particular we obtain some exact sequences of the fuzzy linear maps over the fuzzy quotient spaces.
\end{abstract}

Key Words: Fuzzy $R$-module, Fuzzy linear map(Fuzzy $R$-map), Fuzzy weak isomorphism, Quasi-monic, Epic, Fuzzy quotient spaces.

\section{Introduction}

Fuzzy modules were introduced by Negoita and Ralescu [1]. Katsaras and Liu [2], and Lowen [3] have developed the theory of fuzzy vector spaces.

Fu-Zhen Pan [4] investigated fuzzy vector spaces for the following purposes; to establish a fundamental frame of fuzzy vector space by virtue of homological algebra and modular theory, and to stretch it out to study general fuzzy modules.

In fact, fuzzy vector spaces are the simplest kind of fuzzy free modules. The theory of fuzzy modules has been a virgin field for a long time.

Recently, many authors presented the same research on fuzzy modules, properties of fuzzy finitely generated modules and fuzzy quotient modules, etc.

In particular, Fu-Zhen Pan [5] and Kim [6] investigated the properties of the sequence of fuzzy linear maps and studied the situations in connection with two exact sequences of fuzzy linear maps.

In this paper, we study various properties of fuzzy linear maps over fuzzy quotient spaces.

\section{Preliminaries}

In this section, we review some definitions and some results which will be used in the later sections. Throughout this paper, we assume that all modules are equipped with the same underlying commutative ring $R$.

Manuscript received Feb. 21, 2012; revised Jun. 15, 2012; accepted Jun. $18,2012$.

${ }^{*}$ Corresponding author: Chang Bum Kim(cbkim@kookmin.ac.kr)

This work was supported by the research program 2012 of Kookmin University in Korea.

(c)The Korean Institute of Intelligent Systems. All rights reserved.
Definition 2.1 ([5]). A $R$-module $M$ together with a function $\chi$ from $M$ into $[0,1]$ is called a fuzzy $R$-module if it satisfies the following conditions;

1. $\chi(a+b) \geq \min \{\chi(a), \chi(b)\}$

2. $\chi(-a)=\chi(a)$

3. $\chi(0)=1$

4. $\chi(r a) \geq \chi(a)$,

for any $a, b \in M$ and $r \in R$, denoted by $(M, \chi)$ or $\chi_{M}$.

Definition 2.2 ([5]). Let $\chi_{M}, \eta_{N}$ be any two fuzzy $R$ modules, then $\tilde{f}: \chi_{M} \longrightarrow \eta_{N}$ is called a fuzzy linear map (or fuzzy $R$-map) if there exists a linear map $f: M \longrightarrow N$ such that $\eta(f(a)) \geq \chi(a)$ for all $a \in M$.

Remark 2.3. Let $\chi_{M}, \eta_{N}$ be any two fuzzy $R$-modules. Then $\tilde{f}: \chi_{M} \longrightarrow \eta_{N}$ is called a fuzzy strong linear map if there exists a linear map $f: M \longrightarrow N$ such that $\eta(f(a))=$ $\chi(a)$ for all $a \in M$.

Definition 2.4 ([5]). Let $\tilde{f}: \chi_{V} \longrightarrow \eta_{W}$ be a fuzzy linear map. $\tilde{f}$ is called a fuzzy weak isomorphism if $f$ is an isomorphism, denoted $\chi_{V} \simeq_{W}^{f} \eta_{W}$.

Definition 2.5 ([5]). For a fuzzy linear map $\tilde{f}: \chi_{M} \longrightarrow$ $\eta_{N}, \eta_{\operatorname{Imf}}$ is called the image of $\tilde{f}$ denoted by $\eta_{\operatorname{Im} \tilde{f}}$. Further, $\chi_{M_{0}}$, where $M_{0}=\{m \in M \mid \eta(f(m))=1\}$ is called the Kernel of $\tilde{f}$ denoted by $\chi_{\text {Ker } \tilde{f}}$.

Theorem 2.6 ([5]). Let $\tilde{f}: \chi_{M} \longrightarrow \eta_{N}$ be a fuzzy linear map, then $\chi_{K e r \tilde{f}}$ is a fuzzy subspace of $\chi_{M}$ and $\eta_{I m \tilde{f}}$ is a fuzzy subspace of $\eta_{N}$.

Remark 2.7. For any fuzzy linear map $\tilde{f}: \chi_{M} \longrightarrow \eta_{N}$,

$$
\chi_{K \operatorname{Kerf}} \leq \chi_{K e r \tilde{f}} \text { and } \eta_{I m f}=\eta_{\operatorname{Im} \tilde{f}} .
$$


Definition 2.8 ([5]). A fuzzy linear map $\tilde{f}: \chi_{M} \longrightarrow \eta_{N}$ is called epic (or monic) iff $f: M \longrightarrow N$ is epic (or monic).

Definition 2.9 ([5]). A fuzzy linear map $\tilde{f}: \chi_{M} \longrightarrow \eta_{N}$ is called quasi-monic iff $\chi_{\text {Ker } \tilde{f}}=\chi_{M^{\prime}}$, where $M^{\prime}=\{m \in$ $M \mid \chi(m)=1\}$.

Remark 2.10. Obviously, when $\chi_{\text {Ker } \tilde{f}}=\{0\}$, quasimonic is just ordinary monic.

Definition 2.11 ([5]). Two fuzzy maps

$$
\chi_{M} \stackrel{\tilde{f}}{\rightarrow} \eta_{N} \stackrel{\tilde{g}}{\rightarrow} \rho_{V}
$$

are exact at $\eta_{N}$ iff $\eta_{\text {Im } \tilde{f}}=\eta_{K e r \tilde{g}}$.

Remark 2.12. By the induction, from Definition 2-11, we can define an exact sequence of fuzzy linear maps.

Theorem 2.13 ([5]). A fuzzy $R$-module $\chi_{M}$ is called a fuzzy singular $R$-module iff $\chi(m)=1$ for all $m \in M$, denoted by 1 .

Theorem 2.14 ([5]). An exact sequence

$$
1 \stackrel{\tilde{i}}{\rightarrow} \chi_{M} \stackrel{\tilde{f}}{\rightarrow} \eta_{N} \stackrel{\tilde{g}}{\rightarrow} \rho_{V} \stackrel{\tilde{j}}{\rightarrow} 1
$$

where the two 1's are the appropriate singular fuzzy $R$ modules and $\tilde{i}, \tilde{j}$ are the fuzzy identity map and an epic map, respectively, is called a short exact sequence of fuzzy linear maps.

Theorem 2.15 ([5]). Given a short exact sequence of fuzzy linear maps,

$$
1 \stackrel{\tilde{i}}{\rightarrow} \chi_{M} \stackrel{\tilde{f}}{\rightarrow} \eta_{N} \stackrel{\tilde{g}}{\rightarrow} \rho_{V} \stackrel{\tilde{j}}{\rightarrow} 1
$$

1. $\operatorname{Im} \tilde{i}=\operatorname{Ker} \tilde{f}=\chi_{M^{\prime}}$.

2. $\operatorname{Im} \tilde{f}=K e r \tilde{g} \geq \eta_{N^{\prime}}$.

3. $\tilde{g}$ is epic,

where $M^{\prime}=\{m \in M \mid \chi(m)=1\}$ and $N^{\prime}=\{n \in N \mid$ $\eta(n)=1\}$.

Proposition 2.16 ([3]). For any fuzzy linear map $\tilde{f}$ : $\chi_{M} \longrightarrow \eta_{N}$,

$$
\chi_{M^{\prime}} \subseteq \chi_{K e r \tilde{f}},
$$

where $M^{\prime}=\{m \in M \mid \chi(m)=1\}$.

Definition 2.17 ([8]). By the coimage Coim $h$ and the cokernel Coker $h$ of a homomorpism $h: X \longrightarrow Y$, we mean the quotient modules

$$
\text { Coim } h=X / \text { Kerh, Coker } h=Y / \text { Imh } .
$$

\section{Fuzzy linear maps over fuzzy quotient spaces}

In this section, we study various properties of fuzzy linear maps over fuzzy quotient spaces.

Let $\chi_{W}$ be an arbitrary fuzzy subspace of a fuzzy vector space $\chi_{V}$. Then a map $\bar{\chi}: V / W \longrightarrow[0,1]$ given by

$$
\bar{\chi}(u+W)= \begin{cases}1 & \text { if } v \in W \\ \inf \left\{\chi\left(v^{\prime}\right) \mid v^{\prime} \in v+W\right\} & \text { if } v \notin W\end{cases}
$$

can determine the fuzzy quotient $\operatorname{space}(V / W, \bar{\chi})$, denoted $\chi_{V / W}$ or $\bar{\chi}_{V / W}($ See $[5])$.

Theorem 3.1. Consider the following diagram of fuzzy $R$ maps of $R$-modules :

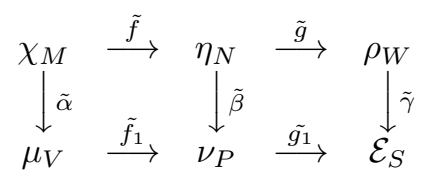

where the rows are exact and the squares are commutative. If $\tilde{\beta}$ is quasi-monic, then the map

$$
\tilde{f}^{*}: \bar{\chi}_{\operatorname{Coim}(\tilde{\alpha})} \longrightarrow \bar{\eta}_{\operatorname{Coim}(\tilde{\beta})}
$$

defined by

$$
\tilde{f} *(m+K e r \tilde{\alpha})=f(m)+\operatorname{Ker} \tilde{\beta}
$$

is a fuzzy linear map.

Proof. First we prove that $\tilde{f}^{*}$ is well-defined. Let $m+$ $\operatorname{Ker} \tilde{\alpha}=m^{\prime}+K e r \tilde{\alpha}$. Then

$$
m=m^{\prime}+x(x \in K e r \tilde{\alpha}) .
$$

Thus

$$
f(m)+\operatorname{Ker} \tilde{\beta}=f\left(m^{\prime}\right)+f(x)+\operatorname{Ker} \tilde{\beta} .
$$

Since

$$
\nu \beta f(x)=\nu f_{1} \alpha(x) \geq \mu \alpha(x)=1,
$$

we have

$$
\nu \beta f(x)=1 .
$$

So

$$
f(x) \in \operatorname{Ker} \tilde{\beta} .
$$

Thus

$$
f(m)+K \operatorname{er} \tilde{\beta}=f\left(m^{\prime}\right)+\operatorname{Ker} \tilde{\beta} .
$$


Thus $\tilde{f} *$ is well-defined. On the other hand,

$$
\begin{aligned}
\tilde{f}^{*}[(m+K e r \tilde{\alpha}) & \left.+\left(m^{\prime}+K e r \tilde{\alpha}\right)\right] \\
& =\tilde{f}^{*}\left(m+m^{\prime}+\operatorname{Ker} \tilde{\alpha}\right) \\
& =f\left(m+m^{\prime}\right)+\operatorname{Ker} \tilde{\beta} \\
& =f(m)+f\left(m^{\prime}\right)+\operatorname{Ker} \tilde{\beta} \\
& =(f(m)+\operatorname{Ker} \tilde{\beta})+\left(f\left(m^{\prime}\right)+\operatorname{Ker} \tilde{\beta}\right) \\
& =\tilde{f}^{*}(m+\operatorname{Ker} \tilde{\alpha})+\tilde{f^{*}}\left(m^{\prime}+\operatorname{Ker} \tilde{\alpha}\right) .
\end{aligned}
$$

For any $r \in R$,

$$
\begin{aligned}
\tilde{f}^{*}(r(m+K e r \tilde{\alpha})) & =\tilde{f} *(r m+K e r \tilde{\alpha}) \\
& =f(r m)+K e r \tilde{\beta} \\
& =r f(m)+K e r \tilde{\beta} \\
& =r[f(m)+K \operatorname{er} \tilde{\beta}] \\
& =r \tilde{f}^{*}(m+K e r \tilde{\alpha}) .
\end{aligned}
$$

Thus $\tilde{f}^{*}$ is a linear map. To prove that $\tilde{f}^{*}$ is a fuzzy linear map, we must show that

$$
\bar{\eta} \tilde{f} *(m+K e r \tilde{\alpha}) \geq \bar{\chi}(m+K e r \tilde{\alpha})
$$

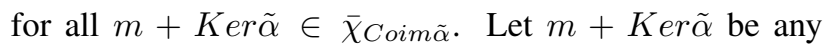
element of $\bar{\chi}_{\text {Coim } \tilde{\alpha}}$. If $m \in \operatorname{Ker} \tilde{\alpha}$, then

$$
\begin{aligned}
\bar{\eta} \tilde{f}^{*}(m+\text { Ker } \tilde{\alpha}) & =\bar{\eta} \tilde{f}^{*}(0) \\
& =\bar{\eta}(0) \\
& =1 \\
& \geq \bar{\chi}(m+\text { Ker } \tilde{\alpha}) .
\end{aligned}
$$

Let $m \notin K e r \tilde{\alpha}$. If $f(m) \in K e r \tilde{\beta}$, then

$$
\begin{aligned}
\bar{\eta} \tilde{f}^{*}(m+K e r \tilde{\alpha}) & =\bar{\eta}(f(m)+K e r \tilde{\beta}) \\
& =1 \\
& \geq \bar{\chi}(m+K e r \tilde{\alpha}) .
\end{aligned}
$$

If $f(m) \notin K \operatorname{Ker} \tilde{\beta}$, then

$$
\begin{aligned}
\bar{\eta} \tilde{f^{*}(m} & +\operatorname{Ker} \tilde{\alpha}) \\
& =\bar{\eta}(f(m)+\operatorname{Ker} \tilde{\beta}) \\
& =\inf \{\eta(x) \mid x \in f(m)+\operatorname{Ker} \tilde{\beta}\} \\
& =\inf \{\eta(f(m)+y) \mid y \in \operatorname{Ker} \tilde{\beta}\} \\
& \geq \inf \{\min \{\eta f(m), \eta(y)\} \mid y \in \operatorname{Ker} \tilde{\beta}\} \\
& \geq \inf \{\min \{\chi(m), \eta(y)\} \mid y \in \operatorname{Ker} \tilde{\beta}\} \\
& =\chi(m) \quad[\tilde{\beta} \text { is quasi-monic }] \\
& \geq \bar{\chi}(m+\operatorname{Ker} \tilde{\alpha}) .
\end{aligned}
$$

Theorem 3.2. Consider the following diagram of fuzzy $R$ maps of $R$-modules :

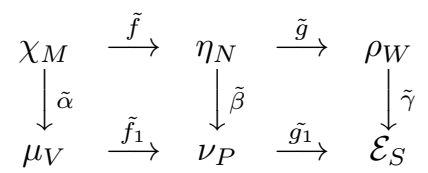

where the rows are exact and the squares are commutative. If $\tilde{\beta}$ and $\tilde{\gamma}$ are quasi-monic, then the following sequence

$$
\bar{\chi}_{\operatorname{Coim}(\alpha)} \stackrel{\tilde{f}^{*}}{\rightarrow} \bar{\eta}_{\operatorname{Coim}(\beta)} \stackrel{\tilde{g}^{*}}{\rightarrow} \bar{\rho}_{\operatorname{Coim}(\gamma)}
$$

is exact.

Proof. By Theorem 3.1, we can construct the fuzzy linear map

defined by

$$
\tilde{g}^{*}: \bar{\eta}_{\operatorname{Coim}(\tilde{\beta})} \longrightarrow \bar{\rho}_{\operatorname{Coim}(\tilde{\gamma})}
$$

$$
\tilde{g}^{*}(n+\operatorname{Ker} \tilde{\beta})=g(n)+\operatorname{Ker} \tilde{\gamma}
$$

To prove that the given sequence in Theorem 3.2 is exact, we must show that

$$
\bar{\eta}_{I m \tilde{f}^{*}}=\bar{\eta}_{K e r \tilde{g}^{*}}
$$

Let $n+\operatorname{Ker} \tilde{\beta}$ be any element of $\operatorname{Im} \tilde{f}^{*}$. Then there exists $m+\operatorname{Ker} \tilde{\alpha} \in \operatorname{Coim}(\tilde{\alpha})$ such that

$$
\tilde{f}^{*}(m+K e r \tilde{\alpha})=n+K \operatorname{er} \tilde{\beta} .
$$

Thus $f(m)+K \operatorname{er} \tilde{\beta}=n+\operatorname{Ker} \tilde{\beta}$ and thus $n=f(m)+$ $b(b \in \operatorname{Ker} \tilde{\beta})$. Since $\tilde{\beta}$ is quasi-monic, $b \in N^{\prime}=\{x \mid$ $\eta(x)=1\}$. So $\eta(b)=1$ and so $\rho g(b)=1$. If $n \in \operatorname{Ker} \tilde{\beta}$, then

$$
\begin{aligned}
\bar{\rho} \tilde{g}^{*}(n+\operatorname{Ker} \tilde{\beta}) & =\bar{\rho} \tilde{g}^{*}(0) \\
& =\bar{\rho}(0) \\
& =1 .
\end{aligned}
$$

Let $n \notin K \operatorname{Ker} \tilde{\beta}$. If $g(n) \in K \operatorname{Ker} \tilde{\gamma}$, then

$$
\begin{aligned}
\bar{\rho} \tilde{g}^{*}(n+\operatorname{Ker} \tilde{\beta}) & =\bar{\rho}(g(n)+\operatorname{Ker} \tilde{\gamma}) \\
& =\bar{\rho}(0) \\
& =1 .
\end{aligned}
$$

Let $g(n) \notin K e r \tilde{\gamma}$. If $z \in K e r \tilde{\gamma}$, then $\mathcal{E} \gamma(z)=1=$ $\rho(z)$, since $\tilde{\gamma}$ is quasi-monic. Also since $\operatorname{Im} \tilde{f}=\operatorname{Ker} \tilde{g}$ and $f(m) \in \operatorname{Im} \tilde{f}, f(m) \in \operatorname{Ker} \tilde{g}$. So $\rho g f(m)=1$. Thus

$$
\begin{aligned}
\bar{\rho} \tilde{g}^{*}(n+\operatorname{Ker} \tilde{\beta}) & =\bar{\rho}(g(n)+\operatorname{Ker} \tilde{\gamma}) \\
& =\inf \{\rho(x) \mid x \in g(n)+\operatorname{Ker} \tilde{\gamma}\} \\
& =\inf \{\rho(g(n)+z) \mid z \in \operatorname{Ker} \tilde{\gamma}\} \\
& \geq \inf \{\min \{\rho g(n), \rho(z)\} \mid z \in \operatorname{Ker} \tilde{\gamma}\} \\
& =\rho g(n) \\
& =1,
\end{aligned}
$$

since $\rho g(n)=\rho g(f(m)+b)=\rho(g f(m)+g(b)) \geq$ $\min \{\rho g f(m), \rho g(b)\}=1$. In any case, we have $\bar{\rho} \tilde{g}^{*}(n+$ $\operatorname{Ker} \tilde{\beta})=1$. Thus $n+\operatorname{Ker} \tilde{\beta} \in \operatorname{Ker} \tilde{g}^{*}$. Hence

$$
\bar{\eta}_{I m \tilde{f}^{*}} \subseteq \bar{\eta}_{K e r \tilde{g}^{*}}
$$


Conversely, if $n+\operatorname{Ker} \tilde{\beta} \in \bar{\eta}_{K e r \tilde{g}^{*}}$, then

$$
\begin{aligned}
\bar{\rho} \tilde{g}^{*}(n+K e r \tilde{\beta}) & =\bar{\rho}(g(n)+K e r \tilde{\gamma}) \\
& =1
\end{aligned}
$$

Thus $g(n) \in K e r \tilde{\gamma}$. For if $g(n) \notin K e r \tilde{\gamma}$, then

$$
\begin{aligned}
1=\bar{\rho} g^{*}(n+\operatorname{Ker} \tilde{\beta}) & =\bar{\rho}(g(n)+\operatorname{Ker} \tilde{\gamma}) \\
& =\inf \{\rho(g(n)+z) \mid z \in \operatorname{Ker} \tilde{\gamma}\} .
\end{aligned}
$$

Thus $\rho g(n)=1$. Since $\mathcal{E} \gamma g(n) \geq \rho g(n)=1, \mathcal{E} \gamma g(n)=$ 1 and thus $g(n) \in \operatorname{Ker} \tilde{\gamma}$. This is a contradiction. Thus $g(n) \in \operatorname{Ker} \tilde{\gamma}$. Since $\tilde{\gamma}$ is quasi-monic, we have $\rho g(n)=$ 1. Thus $n \in \operatorname{Ker} \tilde{g}$. Since $\operatorname{Ker} \tilde{g}=\operatorname{Im} \tilde{f}$, there exists $m \in \chi_{M}$ such that $f(m)=n$. Thus

$$
\begin{aligned}
\tilde{f} *(m+K e r \tilde{\alpha}) & =f(m)+K \operatorname{er} \tilde{\beta} \\
& =n+K \operatorname{Ker} \tilde{\beta} .
\end{aligned}
$$

Thus $n+\operatorname{Ker} \tilde{\beta} \in \bar{\eta}_{I m \tilde{f}^{*}}$. Hence

$$
\bar{\eta}_{K e r g^{*}} \subseteq \bar{\eta}_{I m \tilde{f}^{*}} .
$$

This completes the proof.

Theorem 3.3. Consider the following diagram of fuzzy $R$ maps of $R$-modules :

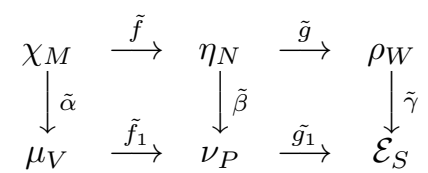

where the rows are exact and the squares are commutative. If $\tilde{f}$ is epic, then the map

$$
{\tilde{f_{1}}}^{*}: \bar{\mu}_{\operatorname{Coker}(\tilde{\alpha})} \longrightarrow \bar{\nu}_{\operatorname{Coker}(\tilde{\beta})}
$$

defined by

$$
\tilde{f_{1}^{*}}(v+\operatorname{Im} \tilde{\alpha})=f_{1}(v)+\operatorname{Im} \tilde{\beta}
$$

is a fuzzy linear map.

Proof. First we prove that $\tilde{f_{1}^{*}}$ is well-defined. Let $v+$ $\operatorname{Im} \tilde{\alpha}=v^{\prime}+\operatorname{Im} \tilde{\alpha}$. Then

$$
v=v^{\prime}+x(x \in \operatorname{Im} \tilde{\alpha}) .
$$

Thus

$$
\begin{aligned}
f_{1}(v)+\operatorname{Im} \tilde{\beta} & =f_{1}\left(v^{\prime}+x\right)+\operatorname{Im} \tilde{\beta} \\
& =f_{1}\left(v^{\prime}\right)+f_{1}(x)+\operatorname{Im} \tilde{\beta}
\end{aligned}
$$

Since $x \in \operatorname{Im} \tilde{\alpha}$, there exists $m \in M$ such that $\tilde{\alpha}(m)=x$. Thus

$$
f_{1}(x)=f_{1} \alpha(m)=\beta f(m) .
$$

So

$$
f_{1}(x) \in \operatorname{Im} \tilde{\beta} .
$$

Thus $f_{1}(v)+\operatorname{Im} \tilde{\beta}=f_{1}\left(v^{\prime}\right)+\operatorname{Im} \tilde{\beta}$. Thus $\tilde{f_{1}^{*}}$ is welldefined. On the other hand,

$$
\begin{aligned}
\tilde{f_{1}}{ }^{*}[(v+\operatorname{Im} \tilde{\alpha}) & \left.+\left(v^{\prime}+\operatorname{Im} \tilde{\alpha}\right)\right] \\
& =\tilde{f_{1}^{*}}\left(v+v^{\prime}+\operatorname{Im} \tilde{\alpha}\right) \\
& =f_{1}\left(v+v^{\prime}\right)+\operatorname{Im} \tilde{\beta} \\
& =f_{1}(v)+f_{1}\left(v^{\prime}\right)+\operatorname{Im} \tilde{\beta} \\
& =\left(f_{1}(v)+\operatorname{Im} \tilde{\beta}\right)+\left(f_{1}\left(v^{\prime}\right)+\operatorname{Im} \tilde{\beta}\right) \\
& =\tilde{f_{1}^{*}}(v+\operatorname{Im} \tilde{\alpha})+\tilde{f_{1}^{*}}\left(v^{\prime}+\operatorname{Im} \tilde{\alpha}\right) .
\end{aligned}
$$

For any $r \in R$,

$$
\begin{aligned}
{\tilde{f_{1}}}^{*}(r(v+\operatorname{Im} \tilde{\alpha})) & =\tilde{f_{1}^{*}}(r v+\operatorname{Im} \tilde{\alpha}) \\
& =f_{1}(r v)+\operatorname{Im} \tilde{\beta} \\
& =r f_{1}(v)+\operatorname{Im} \tilde{\beta} \\
& =r\left[f_{1}(v)+\operatorname{Im} \tilde{\beta}\right] \\
& =r \tilde{f_{1}}{ }^{*}(v+\operatorname{Im} \tilde{\alpha}) .
\end{aligned}
$$

Thus $\tilde{f_{1}}{ }^{*}$ is a linear map. To prove that $\tilde{f_{1}}{ }^{*}$ is a fuzzy linear map, we must show that

$$
\bar{\nu} \tilde{f_{1}^{*}}(v+\operatorname{Im} \tilde{\alpha}) \geq \bar{\mu}(v+\operatorname{Im} \tilde{\alpha})
$$

for all $v+\operatorname{Im} \tilde{\alpha} \in \bar{\mu}_{\text {Coker } \tilde{\alpha}}$. Let $v+\operatorname{Im} \tilde{\alpha}$ be any element of $\bar{\mu}_{\text {Coker } \tilde{\alpha} \text {. Then }}$

$$
\bar{\nu} \tilde{f_{1}^{*}}(v+\operatorname{Im} \tilde{\alpha})=\bar{\nu}\left(f_{1}(v)+\operatorname{Im} \tilde{\beta}\right) .
$$

If $f_{1}(v) \in \operatorname{Im} \tilde{\beta}$, then

$$
\begin{aligned}
\tilde{\nu} \tilde{f_{1}^{*}}(v+\operatorname{Im} \tilde{\alpha}) & =\bar{\nu}\left(f_{1}(v)+\operatorname{Im} \tilde{\beta}\right) \\
& =\bar{\nu}(0) \\
& =1 \\
& \geq \bar{\mu}(v+\operatorname{Im} \tilde{\alpha}) .
\end{aligned}
$$

If $f_{1}(v) \notin \operatorname{Im} \tilde{\beta}$, then

$$
\begin{aligned}
\tilde{\nu}{\tilde{f_{1}}}^{*}(v & +\operatorname{Im} \tilde{\alpha}) \\
& =\bar{\nu}\left(f_{1}(v)+\operatorname{Im} \tilde{\beta}\right) \\
& =\inf \left\{\nu(x) \mid x \in f_{1}(v)+\operatorname{Im} \tilde{\beta}\right\} \\
& =\inf \left\{\nu\left(f_{1}(v)+y\right) \mid y \in \operatorname{Im} \tilde{\beta}\right\} \\
& =\inf \left\{\nu\left(f_{1}(v)+\beta(u)\right) \mid u \in N\right\} \\
& =\inf \left\{\nu\left(f_{1}(v)+\beta f(m)\right) \mid m \in M\right\}[\tilde{f} \text { is epic }] \\
& =\inf \left\{\nu\left(f_{1}(v)+f_{1} \alpha(m)\right) \mid m \in M\right\} \\
& =\inf \left\{\nu\left(f_{1}(v+\alpha(m))\right) \mid m \in M\right\} \\
& \geq \inf \{\mu(v+\alpha(m)) \mid m \in M\} \\
& =\inf \{\mu(v+z) \mid z \in \operatorname{Im} \tilde{\alpha}\} \\
& =\inf \{\mu(x) \mid x \in v+\operatorname{Im} \tilde{\alpha}\} \\
& =\bar{\mu}(v+\operatorname{Im} \tilde{\alpha}) .
\end{aligned}
$$

Hence $\tilde{f_{1}^{*}}$ is a fuzzy linear map. 
Theorem 3.4. Consider the following diagram of fuzzy $R$ maps of $R$-modules :

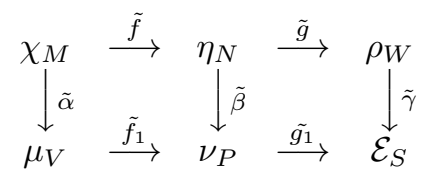

where the rows are exact and the squares are commutative. If $\tilde{f}$ and $\tilde{g}$ are epic, then the following sequence

$$
\bar{\mu}_{\text {Coker }(\tilde{\alpha})} \stackrel{\tilde{f}_{1}^{*}}{\rightarrow} \bar{\nu}_{\operatorname{Coker}(\tilde{\beta})} \stackrel{\tilde{g}_{1}^{*}}{\rightarrow} \overline{\mathcal{E}}_{\operatorname{Coker}(\tilde{\gamma})}
$$

is exact.

Proof. By Theorem 3.3, we can define the fuzzy linear map

$$
\tilde{g}_{1}^{*}: \bar{\nu}_{\operatorname{Coker}(\tilde{\beta})} \longrightarrow \overline{\mathcal{E}}_{\operatorname{Coker}(\tilde{\gamma})}
$$

by

$$
\tilde{g_{1}}{ }^{*}(n+\operatorname{Im} \tilde{\beta})=g_{1}(n)+\operatorname{Im} \tilde{\gamma}
$$

To prove that the given sequence in Theorem 3.4 is exact, we must show that

$$
\bar{\nu}_{I m f_{1}^{*}}=\bar{\nu}_{\operatorname{Kerg}_{1}^{*}} \cdot
$$

Let $p+\operatorname{Im} \tilde{\beta} \in \bar{\nu}_{\operatorname{Kerg}_{1}{ }^{*}}$, then

$$
\begin{aligned}
\overline{\mathcal{E}} \tilde{g_{1}} *(p+\operatorname{Im} \tilde{\beta}) & =\overline{\mathcal{E}}\left(g_{1}(p)+\operatorname{Im} \tilde{\gamma}\right) \\
& =1
\end{aligned}
$$

If $g_{1}(p) \in \operatorname{Im} \tilde{\gamma}$, then there exists $w \in W$ such that $\gamma(w)=$ $g_{1}(p)$. Since $\tilde{g}$ is epic, there exists $n \in N$ such that $g(n)=$ $w$. Thus

$$
\begin{aligned}
g_{1}(p) & =\gamma g(n) \\
& =g_{1} \beta(n) .
\end{aligned}
$$

Thus $p-\beta(n) \in \operatorname{Ker} \tilde{g_{1}}$. Since $\operatorname{Ker} \tilde{g_{1}}=\operatorname{Im} \tilde{f_{1}}$, there exists $v \in V$ such that $f_{1}(v)=p-\beta(n)$. Thus

$$
\begin{aligned}
{\tilde{f_{1}}}^{*}(v+\operatorname{Im} \tilde{\alpha}) & =f_{1}(v)+\operatorname{Im} \tilde{\beta} \\
& =p-\beta(n)+\operatorname{Im} \tilde{\beta} \\
& =p+\operatorname{Im} \tilde{\beta} .
\end{aligned}
$$

Hence

$$
p+\operatorname{Im} \tilde{\beta} \in \bar{\nu}_{I m \tilde{f}_{1}^{*}} .
$$

If $g_{1}(p) \notin I m \tilde{\gamma}$, then

$$
\begin{aligned}
1 & =\overline{\mathcal{E}} g_{1}{ }^{*}(p+\operatorname{Im} \tilde{\beta}) \\
& =\overline{\mathcal{E}}\left(g_{1}(p)+\operatorname{Im} \tilde{\gamma}\right) \\
& =\inf \left\{\mathcal{E}\left(g_{1}(p)+z\right) \mid z \in \operatorname{Im} \tilde{\gamma}\right\} \\
& \leq \mathcal{E} g_{1}(p) .
\end{aligned}
$$

Thus $\mathcal{E} g_{1}(p)=1$. So $p \in \operatorname{Ker} \tilde{g_{1}}$. Since $\operatorname{Ker} \tilde{g_{1}}=\operatorname{Im} \tilde{f}_{1}$, there exists $v \in V$ such that $f_{1}(v)=p$. Thus

$$
\begin{aligned}
{\tilde{f_{1}}}^{*}(v+\operatorname{Im} \tilde{\alpha}) & =f_{1}(v)+\operatorname{Im} \tilde{\beta} \\
& =p+\operatorname{Im} \tilde{\beta} .
\end{aligned}
$$

Hence

and hence

$$
p+\operatorname{Im} \tilde{\beta} \in \bar{\nu}_{I m f_{1}^{*}}
$$

$$
\bar{\nu}_{K e r g_{1}^{*}} \subseteq \bar{\nu}_{I m f_{1}^{*}} \cdot
$$

Conversely let $p+\operatorname{Im} \tilde{\beta}$ be any element of $\bar{\nu}_{\operatorname{Im} f_{1} *}$. Then there exists $v+\operatorname{Im} \tilde{\alpha} \in \bar{\mu}_{\text {Coker }(\tilde{\alpha})}$ such that

$$
\tilde{f_{1}^{*}}(v+\operatorname{Im} \tilde{\alpha})=f_{1}(v)+\operatorname{Im} \tilde{\beta}=p+\operatorname{Im} \tilde{\beta} .
$$

Thus $f_{1}(v)-p \in \operatorname{Im} \tilde{\beta}$. So there exists $n \in N$ such that $\beta(n)=f_{1}(v)-p$. If $g_{1}(p) \in \operatorname{Im} \tilde{\gamma}$, then

$$
\begin{aligned}
\overline{\mathcal{E}} \tilde{g_{1}}{ }^{*}(p+\operatorname{Im} \tilde{\beta}) & =\overline{\mathcal{E}}\left(g_{1}(p)+\operatorname{Im} \tilde{\gamma}\right) \\
& =\overline{\mathcal{E}}(0) \\
& =1 .
\end{aligned}
$$

Let $g_{1}(p) \notin \operatorname{Im} \tilde{\gamma}$. Since $f_{1}(v) \in \operatorname{Im} \tilde{f}_{1}=\operatorname{Ker} \tilde{g_{1}}$, $\mathcal{E} g_{1} f_{1}(v)=1$. Also Since $f_{1} \alpha(m) \in \operatorname{Im} \tilde{f}_{1}=\operatorname{Ker} \tilde{g_{1}}$, $\mathcal{E} g_{1} f_{1} \alpha(m)=1$ for all $m \in M$. Thus we have

$$
\begin{aligned}
\overline{\mathcal{E}} \tilde{g}_{1}^{*}(p+\operatorname{Im} \tilde{\beta}) & =\overline{\mathcal{E}} \tilde{g}_{1}^{*}\left(f_{1}(v)-\beta(n)+\operatorname{Im} \tilde{\beta}\right) \\
& =\overline{\mathcal{E}} g_{1}{ }^{*}\left(f_{1}(v)+\operatorname{Im} \tilde{\beta}\right) \\
& =\overline{\mathcal{E}}\left(g_{1} f_{1}(v)+\operatorname{Im} \tilde{\gamma}\right) \\
& =\inf \left\{\mathcal{E}\left(g_{1} f_{1}(v)+z\right) \mid z \in \operatorname{Im} \tilde{\gamma}\right\} \\
& =\inf \left\{\mathcal{E}\left(g_{1} f_{1}(v)+\gamma(w)\right) \mid w \in W\right\} \\
& \geq \inf \left\{\min \left\{\mathcal{E} g_{1} f_{1}(v), \mathcal{E} \gamma(w)\right\} \mid w \in W\right\} \\
& =\inf \{\mathcal{E} \gamma(w) \mid w \in W\} \\
& =\inf \{\mathcal{E} \gamma g(n) \mid n \in N\}[\tilde{g} \text { is epic }] \\
& =\inf \{\mathcal{E} \gamma g f(m) \mid m \in M\}[\tilde{f} \text { is epic }] \\
& =\inf \left\{\mathcal{E} g_{1} \beta f(m) \mid m \in M\right\} \\
& =\inf \left\{\mathcal{E} g_{1} f_{1} \alpha(m) \mid m \in M\right\} \\
& =1 .
\end{aligned}
$$

Thus

$$
\overline{\mathcal{E}}{\tilde{g_{1}}}^{*}(p+\operatorname{Im} \tilde{\beta})=1
$$

and thus

$$
p+\operatorname{Im} \tilde{\beta} \in \bar{\nu}_{K \operatorname{er} \tilde{g_{1}}}{ }^{*} .
$$

Hence

$$
\bar{\nu}_{I m f_{1}^{*}} \subseteq \bar{\nu}_{\operatorname{Kerg}_{1} *} \cdot
$$

This completes the proof.

Lemma 3.5. Consider the following diagram of fuzzy $R$ maps of $R$-modules :

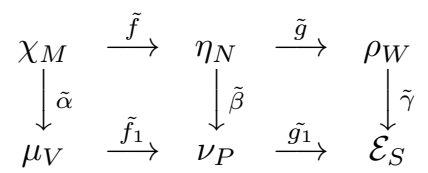


where the rows are exact and the squares are commutative. If $\tilde{g}$ is epic, then for each $w \in \rho_{K e r \tilde{\gamma}}$, there exist $n \in N$ and $v \in V$ such that

$$
g(n)=w \text { and } f_{1}(v)=\beta(n) .
$$

Proof. Let $w$ be any element of $\rho_{K e r \tilde{\gamma}}$. Then $\mathcal{E} \gamma(w)=$ 1. Since $\tilde{g}$ is epic, there exists $n \in N$ such that $g(n)=w$. Thus

$$
\begin{aligned}
\mathcal{E} g_{1} \beta(n) & =\mathcal{E} \gamma g(n) \\
& =\mathcal{E} \gamma(w) \\
& =1 .
\end{aligned}
$$

Thus

$$
\beta(n) \in \operatorname{Ker} \tilde{g_{1}} .
$$

Since $\operatorname{Ker} \tilde{g_{1}}=\operatorname{Im} \tilde{f}_{1}$, we have

$$
\beta(n) \in \operatorname{Im} \tilde{f}_{1} .
$$

Hence there exists $v \in V$ such that $f_{1}(v)=\beta(n)$.

Lemma 3.6. Consider the following diagram of fuzzy $R$ maps of $R$-modules :

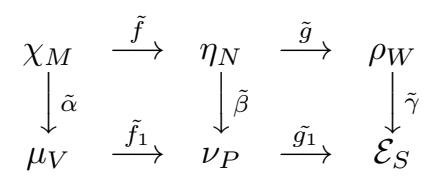

where the rows are exact and the squares are commutative. If $\tilde{f}$ and $\tilde{g}$ are epic, then

1. $\operatorname{Im} \tilde{\beta} \subseteq \operatorname{Im} \tilde{f}_{1}$.

2. $\tilde{f}_{1}(\operatorname{Im} \tilde{\alpha})=\operatorname{Im} \tilde{\beta}$.

Proof. 1. Let $p \in \operatorname{Im} \tilde{\beta}$. Then there exists $n \in \eta_{N}$ such that $\beta(n)=p$. Since $\tilde{f}$ is epic, there exists $m \in \chi_{M}$ such that $f(m)=n$. Thus

$$
p=\beta f(m)=f_{1} \alpha(m) .
$$

So $p \in \operatorname{Im} \tilde{f}_{1}$. Hence $\operatorname{Im} \tilde{\beta} \subseteq \operatorname{Im} \tilde{f}_{1}$.

2. Let $v \in \operatorname{Im} \tilde{\alpha}$. Then there exists $m \in \chi_{M}$ such that $\alpha(m)=v$. Thus

$$
f_{1}(v)=f_{1} \alpha(m)=\beta f(m) .
$$

Hence

$$
\tilde{f}_{1}(\operatorname{Im} \tilde{\alpha}) \subseteq \operatorname{Im} \tilde{\beta} .
$$

On the other hand, let $p \in \operatorname{Im} \tilde{\beta}$. Then there exists $n \in N$ such that $\beta(n)=p$. Since $\tilde{f}$ is epic, there exists $m \in M$ such that $f(m)=n$. Thus $f_{1} \alpha(m)=\beta f(m)=\beta(n)=$ $p$. Hence

$$
p \in f_{1}(\operatorname{Im} \alpha) .
$$

This completes the proof.
Theorem 3.7. Consider the following diagram of fuzzy $R$ maps of $R$-modules :

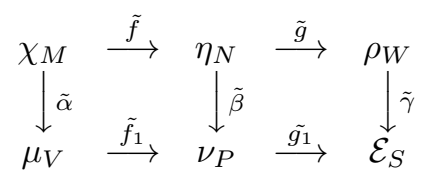

where the rows are exact and the squares are commutative. If $\tilde{f}$ and $\tilde{g}$ are epic, and if $\tilde{f}_{1}$ is monic, then the map

$$
\tilde{h}: \rho_{K e r \tilde{\gamma}} \longrightarrow \bar{\mu}_{\text {Coker } \tilde{\alpha}}
$$

defined by

$$
\tilde{h}(w)=v+\operatorname{Im} \tilde{\alpha},
$$

where $g(n)=w, f_{1}(v)=\beta(n)$ are given by Lemma 3.5, is a zero fuzzy linear map and $\tilde{h}(w)$ does not depend on the choice of $n$ and $v$.

Proof. Let $w$ be any element of $\rho_{K e r \tilde{\gamma}}$ and let $g(n)=$ $w, g\left(n^{\prime}\right)=w, \beta(n)=f_{1}\left((v)\right.$ and $\beta\left(n^{\prime}\right)=f_{1}\left(v^{\prime}\right)\left(n, n^{\prime} \in\right.$ $N$ and $\left.v, v^{\prime} \in V\right)$. Then $h(w)=v+\operatorname{Im} \tilde{\alpha}$ and $h(w)=$ $v^{\prime}+\operatorname{Im} \tilde{\alpha}$, and also $g\left(n-n^{\prime}\right)=0$ and so $n-n^{\prime} \in \operatorname{Ker} \tilde{g}$. Since $\operatorname{Ker} \tilde{g}=\operatorname{Im} \tilde{f}, n-n^{\prime} \in \operatorname{Im} \tilde{f}$. Thus there exists $m \in M$ such that $f(m)=n-n^{\prime}$. Thus

$$
\begin{aligned}
f_{1} \alpha(m) & =\beta f(m) \\
& =\beta\left(n-n^{\prime}\right) \\
& =f_{1}\left(v-v^{\prime}\right)
\end{aligned}
$$

Since $f_{1}$ is monic, $\alpha(m)=v-v^{\prime}$. Thus

$$
\begin{aligned}
v+\operatorname{Im} \tilde{\alpha} & =v^{\prime}+\alpha(m)+\operatorname{Im} \tilde{\alpha} \\
& =v^{\prime}+\operatorname{Im} \tilde{\alpha} .
\end{aligned}
$$

Let $w$ be any element of $\rho_{\text {Ker } \tilde{\gamma}}$ and let $\tilde{h}(w)=v+\operatorname{Im} \tilde{\alpha}$. Then there exist $n \in N$ and $v \in V$ such that $g(n)=w$ and $\beta(n)=f_{1}(v)$. Since $f$ is epic, there exists $m \in M$ such that $f(m)=n$. Thus

$$
\begin{aligned}
f_{1} \alpha(m) & =\beta f(m) \\
& =\beta(n) .
\end{aligned}
$$

Thus

$$
h(w)=\alpha(m)+\operatorname{Im} \tilde{\alpha}=\operatorname{Im} \tilde{\alpha}=0
$$

by definition of $\tilde{h}$. Hence $\tilde{h}$ is a zero map.

Theorem 3.8 ([7]). Consider the following diagram of fuzzy $R$-maps of $R$-modules :

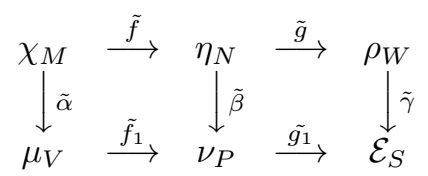


where the rows are exact and the squares are commutative. If $\tilde{f}_{1}: \mu_{V} \longrightarrow \nu_{P}$ is quasi-monic, then the sequence of fuzzy $R$-maps,

$$
\chi_{\text {Ker } \tilde{\alpha}} \stackrel{\tilde{f}^{*}}{\rightarrow} \eta_{\text {Ker } \tilde{\beta}} \stackrel{\tilde{g}^{*}}{\rightarrow} \rho_{\text {Ker } \tilde{\gamma}}
$$

is exact at $\eta_{K e r \tilde{\beta}}$.

From Theorem 3.4 and Theorem 3.8, we have the following theorem.

Theorem 3.9. Consider the following diagram of fuzzy $R$ maps of $R$-modules :

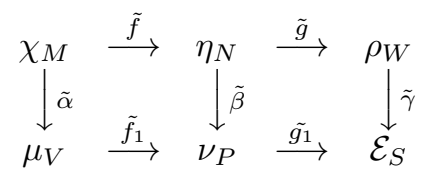

where the rows are exact and the squares are commutative. If $\tilde{f}_{1}$ is monic and if $\tilde{g_{1}}$ is quasi-monic, and if $\tilde{f}$ and $\tilde{g}$ are epic, then the sequence of fuzzy $R$-maps,

$$
\begin{gathered}
\chi_{\text {Ker } \tilde{\alpha}} \stackrel{\tilde{f^{*}}}{\rightarrow} \eta_{\text {Ker } \tilde{\beta}} \stackrel{\tilde{g^{*}}}{\rightarrow} \rho_{\text {Ker } \tilde{\gamma}} \\
\stackrel{\tilde{h}}{\rightarrow} \bar{\mu}_{\text {Coker } \tilde{\alpha}} \stackrel{\tilde{f_{1}^{*}}}{\rightarrow} \bar{\nu}_{\text {Coker }(\beta)} \stackrel{\tilde{g_{1}^{*}}}{\rightarrow} \overline{\mathcal{E}}_{\text {Coker }(\tilde{\gamma})}
\end{gathered}
$$

is exact.

Proof. By Theorem 3.4 and Theorem 3.8, we must show that

$$
\text { (1) } \rho_{I m \tilde{g^{*}}}=\rho_{K \operatorname{Ker} \tilde{h}} \text { and }(2) \bar{\mu}_{I m \tilde{h}}=\bar{\mu}_{K \operatorname{Kerf_{1}^{*}}} \cdot
$$

(1). Let $x$ be any element of $\rho_{I m \tilde{g}^{*}}$. Then there exists $y \in \eta_{K e r \tilde{\beta}}$ such that

$$
\tilde{g^{*}}(y)=g(y)=x .
$$

Since $\tilde{h}$ is a zero map,

$$
\bar{\mu} \tilde{h}(x)=\bar{\mu}(0)=1 .
$$

Thus $x \in \rho_{\text {Ker } \tilde{h}}$. Hence

$$
\rho_{I m g^{*}} \subseteq \rho_{K e r \tilde{h}}
$$

Conversely let $x$ be any element of $\rho_{K e r \tilde{h}}$. Then

$$
\bar{\mu} \tilde{h}(x)=1 .
$$

Since $x \in \rho_{\text {Ker } \tilde{\gamma}}$,

$$
\mathcal{E} \gamma(x)=1
$$

Since $\tilde{g}$ is epic, there exists $y \in \eta_{N}$ such that $g(y)=x$. Since

$$
\mathcal{E} g_{1} \beta(y)=\mathcal{E} \gamma g(y)=1,
$$

we have

$$
\beta(y) \in \nu_{K e r \tilde{g_{1}}}
$$

Since $\tilde{g_{1}}$ is quasi-monic, $\nu_{K e r \tilde{g_{1}}}=\nu_{P^{\prime}}$, where $P^{\prime}=\{x \mid$ $\nu(x)=1\}$. Thus

$$
\nu \beta(y)=1
$$

So

and $g(y)=x$. Thus

$$
y \in \eta_{K e r \tilde{\beta}}
$$

$$
x \in \rho_{\text {Im } \tilde{g}^{*}} .
$$

Hence

$$
\rho_{\text {Ker } \tilde{h}} \subseteq \rho_{\text {Im } \tilde{g}^{*}}
$$

This completes the proof of (1).

(2). To prove (2), we must show that

$$
\bar{\mu}_{K \operatorname{erf} \tilde{1}^{*}}=\{0\}
$$

since $\tilde{h}$ is a zero map. Let $v+\operatorname{Im} \tilde{\alpha}$ be any element of $\bar{\mu}_{\operatorname{Kerf}_{1}{ }^{*}}$. Then

$$
\begin{aligned}
\bar{\nu} \tilde{f_{1}} *(v+\operatorname{Im} \tilde{\alpha}) & =\bar{\nu}\left(f_{1}(v)+\operatorname{Im} \tilde{\beta}\right) \\
& =1 .
\end{aligned}
$$

If $f_{1}(v) \in \operatorname{Im} \tilde{\beta}$, then there exists $n \in N$ such that $\beta(n)=f_{1}(v)$. Since $\tilde{f}$ is epic, there exists $m \in M$ such that $f(m)=n$. Thus

$$
\begin{aligned}
f_{1}(v) & =\beta(n) \\
& =\beta f(m) \\
& =f_{1} \alpha(m) .
\end{aligned}
$$

Since $\tilde{f}_{1}$ is monic, $v=\alpha(m)$. Thus $v+\operatorname{Im} \tilde{\alpha}=\alpha(m)+$ $\operatorname{Im} \tilde{\alpha}=\operatorname{Im} \tilde{\alpha}=0$.

If $f_{1}(v) \notin \operatorname{Im} \tilde{\beta}$, then

$$
\begin{aligned}
1 & =\bar{\nu}{\tilde{f_{1}}}^{*}(v+\operatorname{Im} \tilde{\alpha}) \\
& =\bar{\nu}\left(f_{1}(v)+\operatorname{Im} \tilde{\beta}\right) \\
& =\inf \left\{\nu\left(f_{1}(v)+z\right) \mid z \in \operatorname{Im} \tilde{\beta}\right\} \\
& \leq \nu f_{1}(v) .
\end{aligned}
$$

Thus

$$
\nu f_{1}(v)=1
$$

Thus

$$
v \in \operatorname{Ker} \tilde{f}_{1} .
$$

Since $\tilde{f}_{1}$ is monic, we have $v=0$. Hence

$$
v+\operatorname{Im} \tilde{\alpha}=\operatorname{Im} \tilde{\alpha}=0 .
$$

In any case, we have

$$
v+\operatorname{Im} \tilde{\alpha}=\operatorname{Im} \tilde{\alpha}=0 .
$$

Hence

$$
\bar{\mu}_{\operatorname{Kerf}_{1}^{*}}=\{0\} .
$$

This completes the proof of (2). 


\section{References}

[1] C. V. Negoita and D. A. Ralescu, "Applications of Fuzzy Sets to System Analysis," Birkhäuser, Basel, Chap. 1, pp. 18-24, 1975.

[2] A. K. Katsaras and D. B. Liu, "Fuzzy Vector Spaces and Fuzzy Topological Vector Spaces," J. Math. Anal. Appl., vol. 58, pp. 135-146, 1977.

[3] R. Lowen, "Convex Fuzzy Sets," Fuzzy Sets and Systems, vol. 3, pp. 291-310, 1980.

[4] F. Z. Pan, "Fuzzy Finitely Gernerated Modules," Fuzzy Sets and Systems, vol. 21, pp. 105-113, 1987.

[5] F. Z. Pan, "Exact Sequences of Fuzzy Linear Maps," Fuzzy Sets and Systems, vol. 27, pp. 317-325, 1988.

[6] C. B. Kim, “The Fuzzy Linear Maps,” J. of Fuzzy Logic and Intelligent Systems, vol. 15, no. 4, pp. 511-513, 2005.
[7] C. B. Kim, "A note on the Fuzzy Linear Maps," J. of Fuzzy Logic and Intelligent Systems, vol. 21, no. 4, pp. 506-511, 2011.

[8] S. T. Hu, "Introduction to Homological Algebra," Holden-day, Inc. San Francisco, 1968.

[9] N. G. Northcott, "An Introduction to Homological A1gebra," Cambridge Univ. press, London, 1960.

[10] J. J. Rotman, “An Introduction to Homological Algebra," Academic press, New York, 1972.

\section{Chang Bum Kim}

Professor of Kookmin University

Research Area : Fuzzy Mathematics, Fuzzy Algebra

E-mail : cbkim@kookmin.ac.kr 